\title{
Locomotor activity in juvenile Norway rats as a function of amount of filial huddling at 5-9 days of age
}

\author{
JOSEPH MIELE \\ State University of New York, Albany, New York 12203 \\ and \\ LISA BUDZEK, FRANK COSTANTINI, and RICHARD DENI \\ Rider College, Lawrenceville, New Jersey 08648
}

\begin{abstract}
Norway rat pups (Rattus norvegicus, Long-Evans strain) were studied using a two-phase procedure to determine the effect of amount of filial huddling on later emotionality-activity when tested in a novel apparatus. The infant rats (54 males, 54 females) were raised in litters of reduced size and housed with their dams throughout the study. During Phase 1 of testing, each litter was observed without the dam present to determine the number of different siblings in physical contact with each pup in the huddle clump. Repeated observations were made of pups between 5 and 9 days of age. Total sibling contacts were used as a score representing the amount of filial huddling experienced by each pup. Before Phase 2 began, each pup was assigned, according to sex, to one of three groups ( $n=18$ pups/group), based on the amount of filial huddling experienced in Phase 1: low, medium, and high huddling. During Phase 2, all subjects were tested individually in a shuttle-cage apparatus at 15 and 20 days of age. The frequency of shuttle crossovers was measured and used as an emotionality-activity score. Results indicated that significantly more activity was demonstrated by the high-huddling group. These results are contrasted with data from experimental studies of isolation-induced hyperactivity in juvenile rats and related to general principles of attachment theory.
\end{abstract}

Early social experiences have been implicated in the development of emotionality and responsiveness to novel environments in Norway rats. Certain researchers (Einon \& Morgan, 1978; Randall \& Campbell, 1976) have identified a syndrome of hyperactivity in juvenile rats after periods of housing in social isolation. However, it is unlikely that in the natural environment, juvenile rats would be socially isolated for long periods of time. In fact, brief periods of social contact for these isolatereared juveniles are sufficient to abolish the hyperactivity effect (Einon, Morgan, \& Kibbler, 1978). Other researchers have argued that the hyperactivity effect is the second phase of a two-part isolation effect. According to this view (Dalrymple-Alford \& Benton, 1981), isolates are initially less active and then more active with maturity compared to group-housed rats. This trend for isolates was discussed in terms of their higher levels of fear and greater tendency to remain immobile. Immobility, or "freezing," has remained one of the most common measures of emotionality used by animal behavior researchers. Other measures have recently been reported

Requests for reprints should be addressed to Richard Deni, Department of Psychology, Rider College, Lawrenceville, New Jersey 08648. We thank Debra Hercek, Rene Piper, and Rubin Sanchez for their assistance. in studies of this type, including face washing and grooming. Increases in the frequency of these behaviors have been linked to decreased arousal and fear in novel environments (Archer, 1973). Washing and grooming behaviors in juvenile rats have also been shown to be affected by the degree of the social stimulation during early development. For example, research using litter size as an independent variable representing degree of potential social stimulation has shown that pups from small-sized litters showed less washing and grooming compared with pups from large litters (Akuta, 1979).

The general picture presented by these studies regards restrictions in early social experience as having maladaptive or abnormal consequences on emotionality or responses to novel environments. That restriction may be either severe, as in the case of total social isolation for a period of consecutive weeks, or moderate, as in the case of rearing in a litter of reduced size. The study reported here was an attempt to better define the connection between early social experience and later emotionality. The important change employed in the present study was to classify rat pups according to the amount of filial huddling demonstrated during the 2 nd week of life. Measurement of emotionality occurred on Days 15 and 20 , and differences were tested as a function of amount of filial huddling recorded earlier. The measure 
of emotionality used here was an activity score derived from counting locomotor responses in a shuttle apparatus (Murphy \& Nagy, 1979). Subjects that developed fear in this apparatus would show low activity scores because of the competing fear responses of immobility and freezing. Subjects showing higher activity scores would presumably have habituated more quickly to the novel enviroment and would have demonstrated a lower level of emotionality or fear.

\section{METHOD}

\section{Subjects}

Eighteen Norway rat litters provided 54 male and 54 female pups that were used as subjects. Subjects were male and female offspring obtained from matings of experienced adult male Norway rats with nulliparous adult female Norway rats selected at random from the laboratory colony. Dams were housed with their litters in standard plastic cages with pine shavings as bedding material and with free access to Purina Rat Chow and water. On Day 1 postpartum, each litter was reduced to three male and three female pups by random selection and left undisturbed until Day 5 or 6 postpartum.

\section{Apparatus}

Assessments of the amount of filial huddling were carried out with a given litter located in a clean plastic cage with pine shavings as bedding material. This test cage was positioned under a video camera in a small room apart from the colony room. Both incandescent and fluorescent light sources were present in the test room. Time-sampled video recordings were made during the test session, and the videotapes were coded during playback at a later time.

Assessments of locomotor activity were carried out in a standard rodent shuttle cage (Coulbourn Instruments, Model E10-15). Locomotion from one $16 \times 18 \mathrm{~cm}$ compartment to the next resulted in a switch closure that was counted. The shuttle cage was located inside a sound-attenuating cabinet, which was placed inside a small room apart from the colony room. White masking noise and interior houselights were present inside the apparatus cabinet.

\section{Procedure}

Amount of filial huddling was measured in each subject starting on Day 5 or Day 6 postpartum. Each litter was videotaped during three consecutive daily observation sessions. These sessions took place between 0600 and $1100 \mathrm{~h}$. Prior to the session, each litter was separated from the dam and taken from the home cage. Individual pups were labeled by applying a dotpattern code to the dorsal skin surface using white typing correction fluid. Labeling was required to make individual pup identifications possible during playback of videotapes. The labeled pups were then arbitrarily placed in a clump in the center of a clean test cage and carried to the test room in which videotaping took place. Each taping session lasted $60 \mathrm{~min}$ and consisted of six 2-min episodes separated by $8 \mathrm{~min}$ of elapsed time. A time-sampled record of each litter was made using this procedure. Huddling contact scores were obtained for each of the 108 pups studied by designating each of the 6 pups within a given litter as the "target" pup during a particular playback of the session tape. As each of the six episodes in a given session were played back, an observer recorded the number of different littermates in physical contact with the target. This recording was made only once per episode at the start. Six such recordings constituted the raw data for a given session. The three consecutive daily sessions for each litter were coded in this manner. Huddling contact scores for a given session ranged from 0 to 30 , and the maximum total contacts possible for any subject over all three sessions was 90 . After each videotaping session was com- pleted, the litter was returned to the home cage and dam. During this phase, periodic interobserver reliability was checked, resulting in agreement scores on huddling measures always above $90 \%$.

Male and female subjects were ranked separately based on total number of huddling contacts recorded. Subjects in each of these gender groups were assigned to a low-, medium-, or highhuddling group based on the rankings. Each huddling group consisted of one-third of the sample.

Two assessments of locomotor activity level were carried out in the shuttle-cage apparatus. These sessions occurred at 15 days of age and again at 20 days. Prior to these sessions, all subjects were permanently labeled for identification at 10 days of age by ear punching. Before a given activity test session, a pup was removed from its home cage and carried to the test room in a small clean plastic container. The pup was transferred from the carrying container directly into the shuttle cage, and all cage and cabinet doors were closed. Each activity session lasted $5 \mathrm{~min}$ and an activity score was recorded representing the total number of crossovers from one side of the cage to the other. After the session, the subject was returned to its home cage, the shuttle cage was cleaned, and the pan bedding was changed.

\section{RESULTS}

A three-factor mixed analysis of variance on the locomotor activity scores was done to test for group differences (Kirk, 1968, p. 283). The between-subjects factors were gender and amount of huddling (low, medium, high), and the within-subjects factor was age at activity test $(15,20$ days).

The analysis revealed a significant main effect for amount of huddling $[F(2,48)=3.50, p<.05]$. A nonlinear trend was found for activity scores as a function of amount of huddling, with the highest activity in the high-huddling group and lowest activity in the mediumhuddling group. The group means and standard deviations are shown in Table 1 . The only significant $(p<.05)$

Table 1

Means and Standard Deviations for Locomotion-Activity Scores in Juvenile Rats as a Function of Amount of Filial Huddling, Interaction of Gender and Huddling, Age, and Interaction of Gender and Age

\begin{tabular}{lll}
\hline Group & Mean & SD \\
\hline
\end{tabular}

Main Effect: Amount of Filial Huddling

$\begin{array}{lll}\text { Low Huddling } & 6.1 & 3.3 \\ \text { Medium Huddling } & 5.4 & 3.5 \\ \text { High Huddling } & 6.8 & 3.8\end{array}$

Interaction Effect: Gender by Amount of Huddling $\begin{array}{lll}\text { Males-Low } & 5.3 & 3.2\end{array}$ $\begin{array}{lll}\text { Males-Medium } & 5.4 & 3.8\end{array}$

$\begin{array}{lll}\text { Males-High } & 7.3 & 4.4\end{array}$

Females-Low $\quad 6.8 \quad 3.3$

Females-Medium $\quad 5.3 \quad 3.3$

$\begin{array}{lll}\text { Females-High } & 6.2 & 3.1\end{array}$

Main Effect: Age at Testing

15 Days of Age $\quad 3.3 \quad 2.4$

20 Days of Age $\quad 8.8 \quad 2.0$

Interaction Effect: Gender by Age at Testing

\begin{tabular}{lll} 
Males-15 Days & 2.9 & 2.1 \\
Males-20 Days & 9.1 & 2.3 \\
Females-15 Days & 3.7 & 2.7 \\
Females-20 Days & 8.5 & 1.6 \\
\hline
\end{tabular}


specific comparison according to the Newman-Keuls test was medium vs. high huddling. However, these data do verify that the highest locomotor activity at 15 and 20 days occurred in the group that showed the highest amount of filial huddling at 5-9 days of age.

In addition, there was a significant interaction of gender with amount of huddling $[\mathrm{F}(2,48)=3.20$, $\mathrm{p}=.05]$. There were, however, no significant specific comparisons according to the Newman-Keuls test. The means and standard deviations for the combinations of conditions in the interaction are shown in Table 1. Two trends, differing according to gender, were demonstrated in the interaction. First, the activity scores of male subjects increased as a function of their amount of huddling, and second, activity scores of female subjects declined in a nonlinear way as a function of the huddling variable.

Significantly higher locomotor activity scores were recorded for all subjects at 20 days compared with 15 days $[F(1,48)=223.31, p<.05]$. This main effect of age at testing simply verifies that the subjects locomoted more in an apparatus the second time they were tested in it or that they locomoted more as they matured.

The final significant effect of importance was the interaction of gender with age at activity test $[\mathrm{F}(1,48)=$ $4.26, \mathrm{p}<.05]$. The Newman-Keuls test showed males/ 20 days vs. males/ 15 days and females/20 days vs. females $/ 15$ days to be significant $(p<.05)$. The maturation or retest effect was evident overall and for males and females separately. Group means and standard deviations are shown in Table 1.

\section{DISCUSSION}

Increased locomotor activity has been discussed as evidence of decreased emotionality or arousal in rodents (Archer, 1973). The maturation-retest effect reported here would support this view. However, that position also involves viewing the highhuddling group as the least emotional or aroused, since that group showed significantly higher locomotor activity.

The major implication of these data is that during early development, those offspring that experience higher amounts of social stimulation are less fearful in a novel test apparatus. This position is only partly reinforced by the results of experimental studies using social isolation as an independent variable. For example, Randall and Campbell (1976) reared rat pups (1) alone or (2) with their dam plus three siblings. At 5 and 10 days of age, the two groups did not differ, but at 15 days of age, the isolates exhibited significantly more locomotion than the controls did. The difference was not evident at 20 days of age, however. By contrast, the present data indicate a tendency for pups receiving less sibling contact to be less active in a novel test apparatus. Generally in support of the present data is the report by Dalrymple-Alford and Benton (1981) that suggests that isolation effects must be viewed in two phases. According to Dalrymple-Alford and Benton, the first phase involves isolationinduced immobility and increased fearfulness and the second phase involves isolation-induced arousal. The subjects in that study were tested between 63 and 100 days of age, whereas the sample used in the present research was tested at 15 and 20 days of age. The differential social conditions in the Dalrymple-Alford and Benton study were introduced after weaning at 21 days of age. It is possible that their Phase 1 isolation-induced immobility would be evident in younger pups treated and tested prior to 60 days of age. The present data provide the additional early information on the effects of social stimulation on activity level and do so without exposing the pups to isolation conditions.

The effect of social stimulation on activity and emotionality reported in the present study is further reinforced by general notions concerning mother-offspring attachment. According to general theories of attachment, exploration by the offspring in a novel environment is a reliable predictor of the quality and quantity of mother-offspring interactions (cf. Ainsworth, Blehar, Waters, \& Wall, 1978). The present data are in agreement with those theories and implicate sibling contacts as an integral component. For example, differences in amount of filial huddling observed in the present study represented the number of different littermates in physical contact with a given pup. Under those circumstances, a quantitative factor in sibling attachment was measured, and presumably, the amount of social stimulation experienced by pups during development was related to the filial huddling scores recorded during test sessions. Subjects with high filial huddling scores were the focus of more contacts by separate littermates. What is most encouraging about these data is that they fit well into the pattern of experimental data on social isolation, without involving a treatment so potentially disruptive to other aspects of behavioral development.

\section{REFERENCES}

Ainsworth, M. D. S., Blehar, M. C., Waters, E., \& Wall, S. Patterns of attachment. Hillsdale, N.J: Erlbaum, 1978.

AKUTA, T. Effects of rearing conditions on the behavior of mothers and on the later open-field behavior of the offspring in the mouse. Japanese Journal of Psychology, 1979, 50, 73-81.

Archer, J. Tests for emotionality in rats and mice: $\mathbf{A}$ review. Animal Behavior, 1973, 21, 205-235.

Dalrymple-Alford, J. C., \& Benton, D. Activity differences of individually and group-housed male and female rats. Animal Learning \& Behavior, 1981, 9, 50-55.

Einon, D. F., \& Morgan, M. J. Early isolation produces enduring hyperactivity in the rat, but no effect upon spontaneous alteration. Quarterly Journal of Experimental Psychology, 1978, 30, 151-156.

Einon, D. F., Morgan, M. J., \& Kibblen, C. C. Brief periods of socialization and later behavior in the rat. Developmental Psychobiology, 1978, 11, 213-225.

KIRK, R. E. Experimental design: Procedures for the behavioral sciences. Belmont, Calif: Brooks Cole, 1968.

MuRPhY, J. M., \& NAGY, Z. M. Comparison of behavioral activity in two apparatus for food-deprived mice. Bulletin of the Psychonomic Society, 1979, 14, 43-45.

Randall, P. K., \& Campbell, B. A. Ontogeny of behavioral arousal in rats: Effect of maternal and sibling presence. Journal of Comparative and Physiological Psychology, 1976, 90, 453-455.

(Received for publication July 14, 1982.) 